\title{
The Forgotten, Not Studied or Not Valorized Tricuspid Valve: The Transcatheter Revolution Is Coming
}

\author{
Dinaldo C. Oliveira ${ }^{a, b, c}$, Carolina G.C. Oliveira ${ }^{b}$
}

\begin{abstract}
The tricuspid valve (TV) has been known as the forgotten valve. However, considering recent information from scientific studies, this nomenclature may need to be adjusted for the valve, which also needs to be better studied and understood. For decades, tricuspid regurgitation (TR) was not fully appreciated and was never the priority. However, studies have revealed that such pathology is related to a possible negative impact on prognosis of patients. Severe TR is a predictor of higher mortality. For the treatment of TR, repair or valve replacement can be performed. Repair techniques can be performed on the annulus (suture annuloplasty or ring implant), on the leaflets (e.g. triangular resection), on the cords (transfers or new cords) and on the papillary muscles (e.g. sliding technique). The anatomical characteristics of the TV determine the repair technique to be used. In some cases, valve repair is not possible and/or not indicated and valve replacement is selected based on the strategy. Nowadays transcatheter therapies have been used and studied. The main transcatheter strategies for the treatment of TR are based on reduction of the annulus (Cardioband, Trialign, TriCinch, Millipede and TRAIPTA), improvement of the leaflet coaptation (Mitraclip, FORMA device, PASCAL system, and TV occluder), reduction of the reflux for the vena cava system (Tric valve and Sapien valve implant), and valve implants (Navigate, Trisol, Sapien, Melody). In this context, there are still other devices (such as Tricentro, Pasta, etc.) being developed and tested throughout several phases of research. In the future, improved knowledge of the TV and the evolution of transcatheter treatments will alter the history of the TV. The transcatheter revolution is coming!
\end{abstract}

Keywords: Tricuspid valve; Transcatheter; Tricuspid regurgitation

\section{Introduction}

The tricuspid valve (TV) is the largest of the cardiac valves and the most apical, and is composed of the annulus, leaf-

Manuscript submitted April 22, 2019, accepted June 3, 2019

${ }^{a}$ Federal University of Pernambuco, Recife, Pernambuco, Brazil ${ }^{b}$ Hospital Ilha do Leite, Recife, Pernambuco, Brazil

${ }^{\mathrm{c} C}$ Corresponding Author: Dinaldo C. Oliveira, Federal University of Pernambuco, Recife, Pernambuco 52011-000, Brazil. Email: dinaldo@cardiol.br

doi: https://doi.org/10.14740/cr874 lets, chordae tendineae, and papillary muscles. The velocity of blood through this valve is $1 \mathrm{~mm} / \mathrm{s}$, the gradient is less than or equal to $2 \mathrm{~mm} \mathrm{Hg}$, and its orifice is $7-9 \mathrm{~cm}^{2}$ in size [1].

The annulus has a non-planar D shape and multiple muscle bars from muscle fibers derived from the atrium and the ventricle. Its anterior portion is mainly composed of the right atrium wall and the posterior portion mainly consists of the right ventricle. In addition, it is also formed by the interventricular septum and anterior leaflet. In relation to the sagittal plane, it sits nearly at a 45 -degree angle. It has a dynamic structure and its size can vary by up to $30 \%$ during the cardiac cycle. The largest area of the annulus occurs during atrial contraction and the early phase of diastole $[1,2]$.

There are important cardiac structures close to the annulus that receive special attention during surgical or percutaneous invasive procedures in the TV. The non-coronary aortic valve cusp, the atrioventricular nodule, and the bundle of His are located near the anteroseptal commissure. The coronary sinus is anatomically close to the posterior septal commissure. The right coronary artery is related to the anterior and posterior portions of the annulus $[1,3]$.

Generally, the TV has three leaflets (anterior, medial and septal), but sometimes there are more than those described. The anterior leaflet is the largest, widest, and most mobile leaflet, while the posterior is the shortest in the circumferential plane. The septal leaflet is the shortest in the radial plane and is also the least mobile. As these structures are thinner than the mitral valve leaflets, it is sometimes challenging to perfectly visualize the three leaflets via imaging methods. The anteroseptal commissure is located between the basal portions of the anterior and septal leaflets, the anteroposterior commissure sits between the anterior and posterior leaflets, and the posterior septal commissure is between the septal and posterior leaflets. The coaptation length ranges from $5-10 \mathrm{~mm}$ $[1,4]$.

The tendinous cords are formed by up to $80 \%$ collagen and are numerous, with an average number of 25 (ranging from 17 to 36). There are cords connecting the septum to the anterior and posterior leaflets. There are cords leaving the anterior papillary muscle and connecting the anterior and posterior leaflets, others leaving the posterior papillary muscle (can be bifid or trifid) to the posterior and septal leaflets, and others from the septal papillary muscle to the anterior and septal leaflets. Accessory cords are those connecting the right ventricle free wall to the moderator band, while false cords connect the papillary muscle to the right ventricle free wall. The cord can be fan-shaped, basal, and have a rough zone, free edge, and 


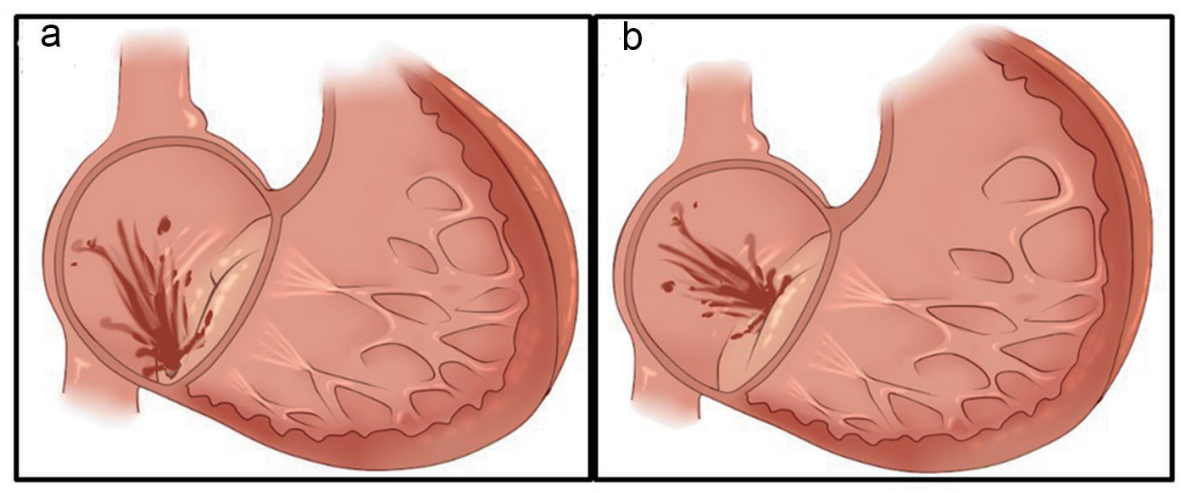

Figure 1. (a) Primary tricuspid regurgitation (structural valve abnormalities). (b) Secondary tricuspid regurgitation (normal valve structures).

deep chordae $[1,2,4]$.

\section{Tricuspid Regurgitation (TR) and Its Clinical Impact}

The TV has been known as the forgotten valve. However, considering recent information from scientific studies, this nomenclature may need to be adjusted for the valve, which also needs to be better studied and understood.

Considering the prevalence of TR in the USA, it is estimated that more than 1.6 million people have TR. A community study that evaluated 16,380 people revealed a prevalence of $0.5 \%$, with a higher frequency in women and those at an older age. In this study, TR was an independent predictor of mortality. It is also noted that the prevalence of atrial fibrillation in those who had TR was high and had an impact on mortality $[5,6]$.

Prihadi et al [7] evaluated 1,000 patients with trace or mild TR and showed the progression rate to be moderate and severe. Older age, implantable intracardiac devices, reduced right ventricle function, a dilated right atrium, and previous left ventricular valve surgery were factors associated with a rapid progression to severe TR. Moreover, the rapid development of severe TR was a factor that increases patient mortality (odds ratio (OR): 0.92, confidence interval (IC): 0.90 - 0.94, $\mathrm{P}<0.001$ ).

For decades, TR was not fully appreciated and was never the priority. However, studies have revealed that such pathology is related to a possible negative impact regarding the prognosis of patients [8].

TR can be primary (PTR) or secondary (STR) [8]. In primary TR, one of the components of the TV apparatus is abnormal and the most frequent causes are infective endocarditis, rheumatic valve disease, myxomatous disease, Ebstein's anomaly, use of drugs for weight loss, trauma, radiation, implantable electronic cardiac devices, carcinoid syndrome, and systemic diseases, such as systemic lupus erythematosus and arrhythmogenic right ventricular dysplasia, among others [7,9].

On the other hand, the TV is normal in secondary TR, but there are changes in the geometry of the right ventricle. The main causes are left ventricular valve diseases, pulmonary hypertension, cardiomyopathy, or any condition that affects RV geometry and function. STR is the most common form of TR, occurring in $75 \%$ of cases. There is usually dilatation of the annulus, followed by changes in RV geometry with displacement of the papillary muscles and leaflet tethering $[3,9,10]$.

Some authors have related long-term atrial fibrillation in the elderly to a third type of TR, which is mainly characterized by the increase of the right atrium and the annulus [9]. Figure 1 shows an illustration of the morphologic types of TR.

A study with 5,223 patients compared the progression of the patients according to TR severity and showed that those with severe regurgitation had lower event-free survival rates when compared to those with no, mild or moderate $(63.9 \%$ vs. $78.9 \%$ vs. $90.3 \%$ vs. $91.7 \%, \mathrm{P}<0.001)$ regurgitation in a follow-up period of $1.4 \pm 1.1$ years compared to those with mild or moderate regurgitation [6].

Topilsky et al carried out a study with people submitted to echocardiogram and demonstrated that the prevalence of TR was higher in women and was associated with age. Isolated TR was an independent predictor of higher mortality (adjusted risk ratio: 1.68; CI: $1.04-2.6, \mathrm{P}=0.03$ ) [11].

Studies have shown that both PTR and STR are associated with increased mortality and hospitalization for heart failure $[4,9,10]$. In addition, a registry that included more than 34,000 transcatheter aortic valve implantation (TAVI)-treated patients showed that the severity of TR prior to the procedure was independently associated with increased mortality rates after 1 year [12].

A meta-analysis that evaluated 2,840 patients showed that TV repair during mitral and aortic valve surgery reduced cardiac mortality. In addition, during the follow-up period, the occurrence of TR that was $\geq$ moderate and TR progression were lower in patients who underwent TV surgery [13].

Therefore, in several clinical settings, both PTR and STR had a negative impact on the prognosis of patients, which defies the classic clinical knowledge prevailing in the past decades wherein severe TR was not adequately valued.

The severity of TR can be assessed by an echocardiogram (two-dimensional, three-dimensional, transthoracic and transesophageal) using qualitative criteria (inferior vena cava size, right atrium size, right ventricle size, ventricular septum motion, TV morphology, color flow jet, jet contour, flow con- 
Table 1. Echocardiographic Criteria for Evaluation of Ttricuspid Regurgitation

\begin{tabular}{lll}
\hline Qualitatitive & Semi-quantitative & Quantitative \\
\hline IVC size & Tricuspid annulus & EROA (by PISA) \\
Right atrium size & Jet area & Regurgitante volume (by PISA) \\
Right ventricle size & Vena contracta & \\
TV morphology & PISA radius & \\
Interventricular septal motion & Hepatic vein flow \\
Color flow TR jet & Tricuspid inflow \\
Flow convergence zone & \\
TR jet contour & & \\
\hline
\end{tabular}

IVC: inferior vena cava; TV: tricuspid valve; TR: tricuspid regurgitation; PISA: proximal isosurface velocity area; EROA: effective regurgitante orifice area.

vergence zone), semi-quantitative criteria (annulus dilation, vena cava width, proximal isovelocity surface area, jet area, hepatic flow, TV inflow), and quantitative criteria (effective regurgitant orifice area, regurgitant volume) (Table 1). Computed tomography (CT) with multiple decoders and magnetic resonance imaging has been used in some cases, which has been called an integrative approach [3, 4, 10, 14]. Figure 2 shows an example of severe TR according to echocardiogram.

The surgical indications for PTR include patients with severe PTR undergoing left-side valve surgery (IC), symptomatic severe RT without right ventricle dysfunction (IC), moderate TR undergoing left-side valve surgery (IIa C), asymptomatic or mild isolated severe TR, or right ventricle dysfunction (IIa C). On the other hand, the indications for STR consist of patients with severe TR undergoing left-side valve surgery (IC), mild or moderate TR with an annulus of $>40 \mathrm{~mm}$ or $>21 \mathrm{~mm}^{2}$ undergoing left-side valve surgery (IIa), mild or moderate TR with heart failure undergoing left-side valve surgery (IIb), or severe and symptomatic TR after left-side valve surgery without right or left ventricle dysfunction or pulmonary arterial hypertension [15].

There is no indication for transcatheter treatment based on data with strong scientific evidence. This aspect is currently being investigated.

\section{Surgical Techniques for the Treatment of Tricus- pid Insufficiency}

For the treatment of TR, repair or valve replacement can be performed. Repair techniques can be performed on the annulus (suture annuloplasty or ring implant), on the leaflets (e.g. triangular resection), on the cords (transfers or new cords) and on the papillary muscles (e.g. sliding technique). The anatomical characteristics of the TV determine the repair technique to be used. In some cases, valve repair is not possible and/or not indicated and valve replacement is selected based on the strategy [16].

The Kay procedure consists of an annuloplasty through bicuspidization of the TV using a suture placed through the posterior leaflet and the commissures, with exclusion of the posterior leaflet, while the De Vega plasty is performed with a double suture parallel with the posterior septal commissure and the anteroseptal commissure $[17,18]$.

Rings used for annuloplasty can be incomplete, rigid, or semi-rigid, as studies have shown better results with these types of rings. Routinely, a reduction of the annulus is performed. Compared to flexible rings, rigid or semi-rigid rings show lower valve regurgitation rates and higher dehiscence rates during the follow-up period, but such dehiscence has no significant clinical consequences [19].

Studies have identified factors associated with increased chances of recurrence of regurgitation after plasty with rings. The left ventricle ejection fraction (LVEF), tethering height and tethering area in the preoperative period were identified according to univariate analysis, but only tethering height was found to be a factor according to multivariate analysis. In the early postoperative period, LVEF and TR severity were predictors according to univariate and multivariate analyses [20, 21].
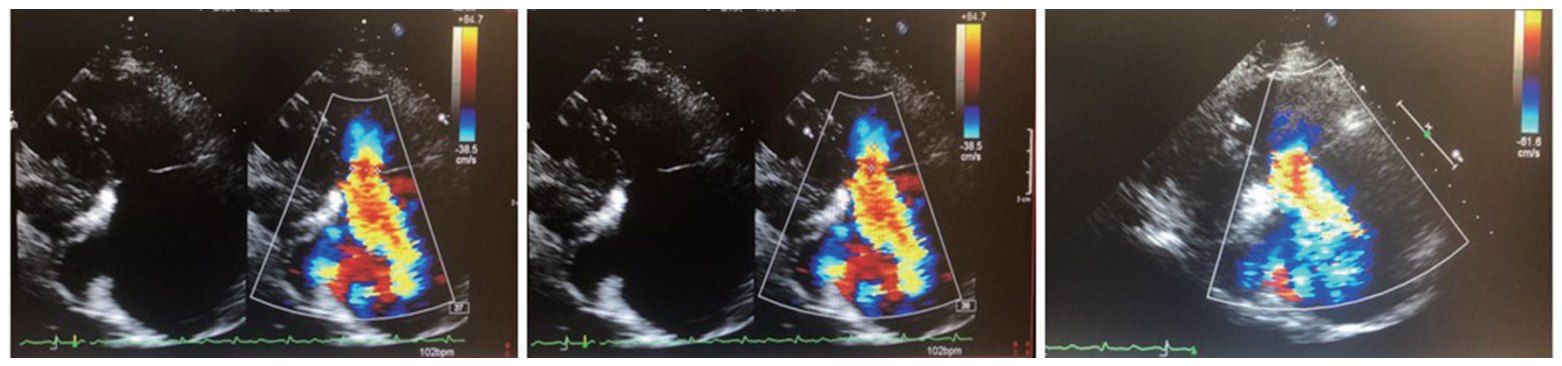

Figure 2. Severe tricuspid regurgitation (effective regurgitante orifice area by PISA $=65 \mathrm{~mm}^{2}$ ). PISA: proximal isosurface velocity area. 
The annuloplasty technique with rigid or semi-rigid incomplete ring implants showed better results compared to sutures and is the most selected technique for annuloplasty. In some cases, the annuloplasty is not sufficient; therefore, it is necessary to increase the anterior leaflet with pericardium or the Clover technique (stitching together the middle point of the free edges of the tricuspid leaflets) [16, 19].

In some situations, cord transfers, new cord implants, triangular resection, and sliding techniques for papillary muscle treatment can be performed. These surgical techniques are most commonly applied to treat PTR [16].

Valve replacement can be performed using either a biological or mechanical prosthesis. For the location of the TV, biological prostheses have great durability and low degeneration. Moreover, since oral anticoagulation is not required, it is an appealing option for most patients. On the other hand, in patients $\leq 40$ years old, mechanical prostheses are an interesting alternative $[15,16,22]$.

The decision of repair or valve replacement in the context of TR depends on several factors, some related to the underlying disease, others to the anatomical characteristics of the valve, and others related to patients. Therefore, treatment should be customized and determined by the institution's heart valve team.

Right ventricular dysfunction and elevated systolic pulmonary pressure have been associated with poor outcomes after procedures involving the TV. It is believed that there may be a point at which right ventricular dysfunction is no longer reversible, and thus, invasive treatment of tricuspid pathology no longer has a benefit [23].

Therefore, the intervention in TR should be more precocious. However, for that, we should improve our knowledge on some aspects of this disease, which are currently being and will continue to be investigated in the near future.

\section{Transcatheter Therapy for the Treatment of TR}

The main transcatheter strategies for the treatment of TR are based on reduction of the annulus (Cardioband, Trialign, TriCinch, Millipede and TRAIPTA), improvement of the leaflet coaptation (Mitraclip, FORMA device, PASCAL system, and TV occluder), reduction of the reflux for the vena cava system (Tric valve and Sapien valve implant), and valve implants (Navigate, Trisol, Sapien, Melody). In this context, there are still other devices (such as Tricentro, Pasta, etc.) being developed and tested throughout several phases of research (Fig. 3) $[4,24]$.

The Cardioband received the CE mark in 2018 for the treatment of TR. This device is a polyester sleeve with radiopaque markers spaced $8 \mathrm{~mm}$ apart containing a premounted wire connected to an adjustable spool. Anchors are implanted in the annulus through the sleeve and then the system is adjusted by reducing the dimensions of the annulus. The procedure is guided by fluoroscopy and echocardiography, and angiography of the right coronary artery is routinely performed with special attention to the artery to prevent any injury. This system was initially developed for the mitral valve, and even-

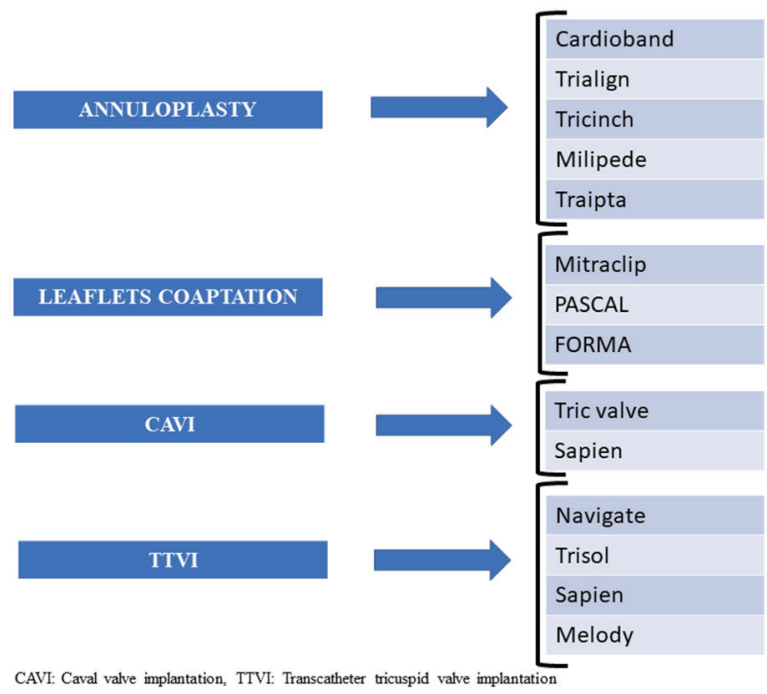

Figure 3. Transcatheter tricuspid valve therapies.

tually the large size of the tricuspid annulus may represent a challenge for adequate annuloplasty [24, 25].

The Tri-Repair (Tricuspid Repair system FIH) study [26] recruited 30 patients with severe TR for Cardioband annuloplasty, and $100 \%$ technical success was achieved. The results after 30 days showed: mortality in $6.7 \%$, stroke in $3.3 \%$, bleeding complications in $13.3 \%$, and coronary complications in $10 \%$. It should be noted that $77 \%$ of the patients did not have adverse clinical events. There was a significant reduction in the septolateral diameter after the procedure (baseline $=43.3 \mathrm{~mm}$ and discharge $=36.9 \mathrm{~mm}, \mathrm{P}<0.001)$, which was maintained at the 6-month follow-up $(36.9 \mathrm{~mm}$ to $37.3 \mathrm{~mm}, \mathrm{P}=\mathrm{NS})$. In the 6-month follow-up assessment, the effective regurgitante orifice area (EROA) was lower than at the baseline $(0.76 \pm$ $0.46 \mathrm{~cm}^{2}$ to $0.39 \pm 0.25 \mathrm{~cm}^{2}, \mathrm{P}<0.01$ ), the New York Heart Association (NYHA) I/II was higher $(20-88 \%, \mathrm{P}<0.01)$, and the quality of life score increased ( $46-70, \mathrm{P}<0.01)$.

The Trialign system was created to perform bicuspidization of the TV, and is placed through the jugular vein using a deflectable radiofrequency catheter positioned below the anteroposterior commissure in the face of the right ventricle and by radio frequency advancing through the annulus until the right atrium where a guidewire is placed, followed by a pledget delivery catheter and later a pledget. The procedure is repeated below the septal-posterior commissure. Using the plication lock device, the two sutures are tightened with annulus plication. It was initially tested in 20 patients with a success rate of 90\% [4]. Subsequently, the Percutaneous Tricuspid Valve Annuloplasty System for Symptomatic Chronic Functional Tricuspid Regurgitation (SCOUT) trial [27] was conducted and 15 patients were evaluated, showing the safety of the device through reduction of the TV diameter $\left(12.3 \mathrm{~mm} \pm 3.1 \mathrm{~cm}^{2}\right.$ to $11.3 \pm 2.7 \mathrm{~cm}^{2}, \mathrm{P}=0.01$ ), reduction of the regurgitant orifice area $\left(0.51 \pm 0.18 \mathrm{~cm}^{2}\right.$ to $\left.0.32 \pm 0.18 \mathrm{~cm}^{2}, \mathrm{P}=0.02\right)$, and efficacy through improvement in NYHA functional class $(\geq$ one class, $\mathrm{P}=0.001)$, and a higher quality of life (47.4 \pm 17.6 to $20.9 \pm 14.8, \mathrm{P}<0.001)$. 
The TriCinch is composed of a corkscrew anchor, selfexpandable stent, and a Dacron band that connects them. Through the femoral vein, using a steerable catheter, the corkscrew anchor is placed inside the anteroposterior portion of the anulus. Subsequently, the self-expandable stent is implanted into the inferior vena cava so there is a cinching (reduction of the septolateral diameter) of the annulus through the Dacron band. A 0.014-inch wire is placed in the right coronary artery at the start of the procedure and an angiography of this coronary artery is performed at the end due to the proximity to the anulus. After performing a first-in-man study, data from the Percutaneous Treatment of Tricuspid Valve Regurgitation With the TriCinch System (PREVENT) study demonstrated technical success in $81 \%$ of patients and the reduction of at least one instance of TR in $94 \%$ of patients $[4,28]$.

Millipede is a complete, semi-rigid ring that has a zig-zag design with eight helical stainless-steel anchors that anchor directly into the annulus. There are eight tensioning sliders that can be used to reduce the annulus to the desired diameter. This device is under investigation [29].

The Traipta device is used to perform an indirect annuloplasty and is formed using a nitinol wire and is pre-shaped into a self-expandable loop to encircle the heart from within the pericardial space. Through the femoral vein, the right atrium is reached, and a guidewire is used to perforate the right atrial appendage and reach the pericardial space. Subsequently, the nitinol loop is positioned in the atrioventricular groove and is tightened. Subsequently, the left atrial appendage is closed with an atrial septal occluder. This device is under investigation $[4,30]$.

The Mitralclip is a device approved for use in mitral valve, being an edge to edge repair because its arms grasp the free edges of the leaflets. The procedure is guided by fluoroscopy and echocardiography. It has been indicated in patients with mitral insufficiency who have a high risk for surgery or who are inoperable or are refractory to the optimized clinical treatment. There are studies that show its use for the treatment of TR in patients with special situations $[4,15,31]$.

Nickening et al [32] treated 64 patients with severe TR who were not candidates for surgery, with Mitraclip, with access through the femoral vein in $89 \%$ of cases and through the internal jugular vein in $11 \%$. There was a 97\% success rate, with improvement in the functional class and the 6-minute walk test findings. Due to the size of the annulus, performing the initial grasp at the anteroseptal commissure was an effective strategy and facilitated the placement of a second central clip.

The Trial to Evaluate Treatment With Transcatheter Clip Repair System in Patients With Moderate or Greater Tricuspid Regurgitation (TRILUMINATE) enrolled 85 patients with moderate or greater TR and no indication for left-sided/pulmonary valve correction was included in the study. The implant success rate and the acute device success rate in this very early study of this very novel treatment were $100 \%$. At 30 days, $86.6 \%$ of patients had at least a one-grade improvement in TR, with the study meeting the primary effectiveness endpoint [33].

The FORMA device is a coaptation facilitator and comprises a spacer (a foam polymer filled balloon that has eight holes for expansion and contains radiolabels) and a rail. Through the subclavian vein, the rail is anchored (distal anchor) and fixed at the apex of the right ventricle. Subsequently, the spacer is positioned with the help of the radiopaque markers in the plane of the TV and the rail is locked in the subclavian and the eventual extra length of the rail is placed in a subcutaneous bag. This system was used in seven patients with a reduction in TR severity and improvement of the patients' quality of life and functional class. A feasibility study is ongoing [4].

The PASCAL system has broad paddles and a central spacer. The leaflets can be captured together or independently. The central spacer is designed to fill the regurgitant orifice area. This device was studied for the treatment of mitral regurgitation and gained a CE mark in 2019. Some clinicians have used it for the treatment of TR according to the compassionateuse program with promising results [4, 34, 35].

To reduce reflux in systemic venous circulation, valves are implanted in the cava veins. The Tric valve is composed of two self-expanding valves that have three leaflets of bovine pericardium. This valve is anchored in the cava atrium junction and its fixation is based on stent design, radial force, and oversizing during implantation. This valve has already been implanted in five patients under compassionate use program. Implants of balloon expandable valves, like the Sapien valves, can be done. Initially, a self-expanding stent should be implanted in the inferior vena cava and this stent should remain $5 \mathrm{~mm}$ inside the right atrium. Then, the balloon valve is implanted above the first hepatic vein using the stent to anchor. For twovalve implants, it is recommended to implant it in the inferior vena cava first and then in the superior vena cava. These valves were implanted in 10 patients in a compassionate-use program and there is a larger ongoing study [4, 24].

The navigate valve is composed of stent (nitinol), three leaflets of equine pericardium, a skirt, twenty-nine grasps that will fix the leaflets on the side of the RV and 12 winglets composed of polyester to seal the right atrium. To implant the valve, a right mini-thoracotomy is performed and through the transatrial route, one guidewire is positioned at the apex, followed by a pigtail through which an extra stiff wire is placed at the apex, and then the pigtail is removed. Subsequently, the introducer is advanced up to 2 to $3 \mathrm{~cm}$ inside the RV. The delivery system is then advanced, and the valve is exposed until the leaflets are reached, followed by atrial implant. After the valve is released, the delivery system is removed, hemostasis is re-established, and the thorax is closed [24, 36].

The International Multicenter Trivalve Registry [37] evaluated 106 patients with RT and heart failure, right ventricular dysfunction, or other severe comorbidities. The success of the procedure was considered when the device was implanted, the delivery system was withdrawn, TR was $\leq 2$ plus, and the patient was alive. Most patients (55\%) were treated with Mitraclips.

The results at 30 days showed that the procedure was a success in $62 \%$ of patients, and there was an improvement of the functional class of heart failure (NYHA), reduction of the TR severity, and mortality of 3\%. At that stage, both feasibility and safety were demonstrated, but suboptimal results were obtained in some cases [37].

Taramasso et al [38] published the results of a 1-year clinical follow-up of this registry, now with 312 patients. The prev- 

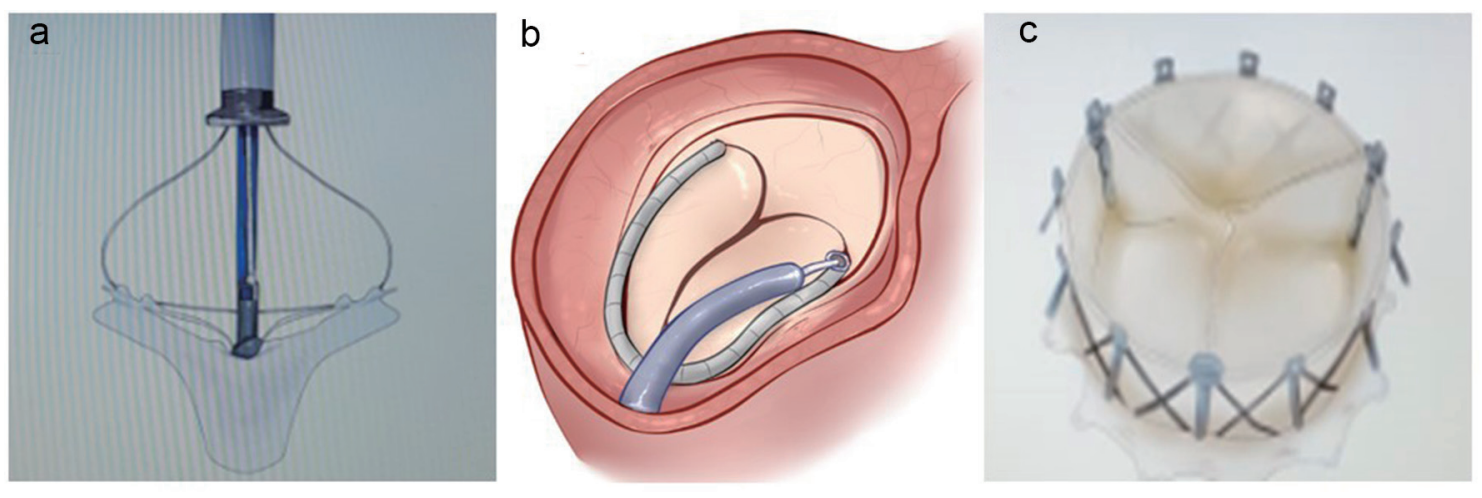

Figure 4. (a) Mitra clip (leaflet grasp). (b) Cardioband (annuloplasty). (c) Navigate (valve).

alence of STR was $92 \%$, the majority of the patients evaluated were women, and the prevalence of atrial fibrillation was $78 \%$. The procedure had a success rate of $72 \%$ (the device was implanted, and the delivery system was withdrawn in all patients, and $\mathrm{TR} \geq 2$ plus was the major contributor to this success rate). The devices used were: Mitraclip (66\%), cava valve implantations (CAVI) (9\%), FORMA (8\%), Trialign (6\%), TriCinch (4\%), Cardioband (4\%), and others $(3 \%)$.

In the Valve-in-Valve International Database (VIVID) registry [39], 152 patients who received expandable balloon valves (Melody: 62\% and Sapien: 38\%) for the treatment of severe tricuspid prosthesis dysfunction were evaluated. Access was gained through the jugular and the procedure had a success rate of $99 \%$. The size of the prostheses was known and the size of the ring was measured by computed tomography (CT-ID). The best projection to work on fluoroscopy and the angle perpendicular to the plane of the tricuspid were defined according to the CT findings. An extra stiff guidewire was positioned within the RV and the delivery system was advanced, and then the valve was released, with the procedure being guided by fluoroscopy and echocardiography without the need for rapid pacing.

During follow-up (median 13.3 months), 22 patients died. There were 10 TV re-interventions and three patients with significant recurrent TV dysfunction. At follow-up, 77\% of patients were in NYHA class I or II $(\mathrm{P}<0.001$ vs. pre tricuspid valve-in-valve (TVIV)). Estimated survival free from TVIV re-intervention and survival free from re-intervention or TVIV dysfunction were $85 \pm 3 \%$ and $83 \pm 3 \%$ at 1 year, respectively. There were no differences in TVIV-related outcomes between Melody and Sapien cohorts [39].

Figure 4 shows some examples of some transcatheter devices for tricuspid intervention.

\section{Conclusions}

TR is not a benign disease. In contrast, when it is severe, in some clinical settings, it is associated with an increase in the occurrence of cardiovascular events, including death.

Knowledge about this valve and its diseases has been improved and classic concepts have been challenged. However, it is still crucial to improve our understanding of the clinical manifestations, diagnosis, treatment, and prognosis of TR.

The transcatheter therapy for the treatment of severe TR has been the focus of the scientific community, industry and society among others. Procedures that are less invasive, safe, and have good short- and long-term results are the aims of this therapy. Some transcatheter devices have shown promising results.

In the future, improved knowledge of the TV and the evolution of transcatheter treatments will alter the history of the TR. The transcatheter revolution is coming.

\section{Acknowledgments}

We would like to thank CAS team (Zurich).

\section{Financial Disclosure}

None.

\section{Conflict of Interest}

None.

\section{Author Contributions}

DCO contributed to searching for articles, writing main text, and producing references, figures, and tables; CGCO searched for articles and corrected references.

\section{References}

1. Dahou A, Levin D, Reisman M, Hahn RT. Anatomy and physiology of the tricuspid valve. JACC Cardiovasc Imaging. 2019;12(3):458-468.

2. Taramasso M, Pozzoli A, Basso C, Thiene G, Denti P, Kuwata S, Nietlispach F, et al. Compare and contrast tricuspid and mitral valve anatomy: interventional perspectives 
for transcatheter tricuspid valve therapies. EuroIntervention. 2018;13(16):1889-1898.

3. Khalique OK, Cavalcante JL, Shah D, Guta AC, Zhan Y, Piazza N, Muraru D. Multimodality Imaging of the Tricuspid Valve and Right Heart Anatomy. JACC Cardiovasc Imaging. 2019;12(3):516-531.

4. Rodes-Cabau J, Hahn RT, Latib A, Laule M, Lauten A, Maisano F, Schofer J, et al. Transcatheter Therapies for Treating Tricuspid Regurgitation. J Am Coll Cardiol. 2016;67(15):1829-1845.

5. Stuge O, Liddicoat J. Emerging opportunities for cardiac surgeons within structural heart disease. J Thorac Cardiovasc Surg. 2006;132(6):1258-1261.

6. Nath J, Foster E, Heidenreich PA. Impact of tricuspid regurgitation on long-term survival. J Am Coll Cardiol. 2004;43(3):405-409.

7. Prihadi EA, Delgado V, Leon MB, Enriquez-Sarano M, Topilsky Y, Bax JJ. Morphologic types of tricuspid regurgitation: characteristics and prognostic implications. JACC Cardiovasc Imaging. 2019;12(3):491-499.

8. Zack CJ, Fender EA, Chandrashekar P, Reddy YNV, Bennett CE, Stulak JM, Miller VM, et al. National trends and outcomes in isolated tricuspid valve surgery. J Am Coll Cardiol. 2017;70(24):2953-2960.

9. Chang JD, Manning WJ, Ebrille E, Zimetbaum PJ. Tricuspid valve dysfunction following pacemaker or cardioverter-defibrillator implantation. J Am Coll Cardiol. 2017;69(18):2331-2341.

10. Hahn RT, Thomas JD, Khalique OK, Cavalcante JL, Praz $\mathrm{F}$, Zoghbi WA. Imaging assessment of tricuspid regurgitation severity. JACC Cardiovasc Imaging. 2019;12(3):469490.

11. Topilsky Y, Maltais S, Medina Inojosa J, Oguz D, Michelena H, Maalouf J, Mahoney DW, et al. Burden of tricuspid regurgitation in patients diagnosed in the community setting. JACC Cardiovasc Imaging. 2019;12(3):433-442.

12. McCarthy FH, Vemulapalli S, Li Z, Thourani V, Matsouaka RA, Desai ND, Kirtane A, et al. Association of tricuspid regurgitation with transcatheter aortic valve replacement outcomes: a report from the society of Thoracic Surgeons/American College of Cardiology Transcatheter Valve Therapy Registry. Ann Thorac Surg. 2018;105(4):1121-1128.

13. Pagnesi M, Montalto C, Mangieri A, Agricola E, Puri R, Chiarito M, Ancona MB, et al. Tricuspid annuloplasty versus a conservative approach in patients with functional tricuspid regurgitation undergoing left-sided heart valve surgery: A study-level meta-analysis. Int J Cardiol. 2017;240:138-144.

14. Rodes-Cabau J, Taramasso M, O'Gara PT. Diagnosis and treatment of tricuspid valve disease: current and future perspectives. Lancet. 2016;388(10058):2431-2442.

15. Ponikowski P, Voors AA, Anker SD, Bueno H, Cleland JGF, Coats AJS, Falk V, et al. 2016 ESC Guidelines for the diagnosis and treatment of acute and chronic heart failure: The Task Force for the diagnosis and treatment of acute and chronic heart failure of the European Society of Cardiology (ESC)Developed with the special contribution of the Heart Failure Association (HFA) of the ESC.
Eur Heart J. 2016;37(27):2129-2200.

16. Belluschi I, Del Forno B, Lapenna E, Nisi T, Iaci G, Ferrara $\mathrm{D}$, Castiglioni A, et al. Surgical techniques for tricuspid valve disease. Front Cardiovasc Med. 2018;5:118.

17. Kay JH, Maselli-Campagna G, Tsuji KK. Surgical Treatment of Tricuspid Insufficiency. Ann Surg. 1965;162:5358.

18. De Vega NG. [Selective, adjustable and permanent annuloplasty. An original technic for the treatment of tricuspid insufficiency]. Rev Esp Cardiol. 1972;25(6):555-556.

19. Pfannmuller B, Davierwala P, Hirnle G, Borger MA, Misfeld M, Garbade J, Seeburger J, et al. Concomitant tricuspid valve repair in patients with minimally invasive mitral valve surgery. Ann Cardiothorac Surg. 2013;2(6):758764.

20. Fukuda S, Gillinov AM, McCarthy PM, Stewart WJ, Song JM, Kihara T, Daimon M, et al. Determinants of recurrent or residual functional tricuspid regurgitation after tricuspid annuloplasty. Circulation. 2006;114(1 Suppl):I582-587.

21. Fukuda S, Song JM, Gillinov AM, McCarthy PM, Daimon M, Kongsaerepong V, Thomas JD, et al. Tricuspid valve tethering predicts residual tricuspid regurgitation after tricuspid annuloplasty. Circulation. 2005;111(8):975-979.

22. Connolly HM, Schaff HV, Abel MD, Rubin J, Askew JW, Li Z, Inda JJ, et al. Early and late outcomes of surgical treatment in carcinoid heart disease. J Am Coll Cardiol. 2015;66(20):2189-2196.

23. Chikwe J, Itagaki S, Anyanwu A, Adams DH. Impact of concomitant tricuspid annuloplasty on tricuspid regurgitation, right ventricular function, and pulmonary artery hypertension after repair of mitral valve prolapse. J Am Coll Cardiol. 2015;65(18):1931-1938.

24. Hahn RT, Nabauer M, Zuber M, Nazif TM, Hausleiter J, Taramasso M, Pozzoli A, et al. Intraprocedural imaging of transcatheter tricuspid valve interventions. JACC Cardiovasc Imaging. 2019;12(3):532-553.

25. Ferrero Guadagnoli A, De Carlo C, Maisano F, Ho E, Saccocci M, Cuevas O, Luciani M, et al. Cardioband system as a treatment for functional mitral regurgitation. Expert Rev Med Devices. 2018;15(6):415-421.

26. Nickenig G, Weber M, Schueler R, Hausleiter J, Nabauer M, von Bardeleben RS, Sotiriou E, et al. 6-month outcomes of tricuspid valve reconstruction for patients with severe tricuspid regurgitation. J Am Coll Cardiol. 2019;73(15):1905-1915.

27. Hahn RT, Meduri CU, Davidson CJ, Lim S, Nazif TM, Ricciardi MJ, Rajagopal V, et al. Early feasibility study of a transcatheter tricuspid valve annuloplasty: SCOUT trial 30-day results. J Am Coll Cardiol. 2017;69(14):17951806.

28. Denti P. EuroPCR 2017, Paris.

29. Rogers JH, Boyd WD, Smith TWR, Ebner AA, Bolling SF. Combined MitraClip Edge-to-Edge repair with millipede IRIS mitral annuloplasty. JACC Cardiovasc Interv. 2018;11(3):323-324.

30. Rogers T, Ratnayaka K, Sonmez M, Franson DN, Schenke WH, Mazal JR, Kocaturk O, et al. Transatrial intrapericardial tricuspid annuloplasty. JACC Cardiovasc 
Interv. 2015;8(3):483-491.

31. Stone GW, Lindenfeld J, Abraham WT, Kar S, Lim DS, Mishell JM, Whisenant B, et al. Transcatheter mitralvalve repair in patients with heart failure. $\mathrm{N}$ Engl J Med. 2018;379(24):2307-2318.

32. Nickenig G, Kowalski M, Hausleiter J, Braun D, Schofer J, Yzeiraj E, Rudolph V, et al. Transcatheter treatment of severe tricuspid regurgitation with the Edge-to-Edge MitraClip technique. Circulation. 2017;135(19):1802-1814.

33. Nickenig G, Lurz P, von Bardeleben S, Sorajja P, Hausleiter J, Denti P, et al. Percutaneous edge-to-edge repair for tricuspid regurgitation: primary outcomes from the TRILUMINATE clinical trial. Presented at: EuroPCR 2019. May 21, 2019. Paris, France.

34. Grasso C, Popolo Rubbio A. The PASCAL transcatheter mitral valve repair system for the treatment of mitral regurgitation: another piece to the puzzle of edge-to-edge technique. J Thorac Dis. 2017;9(12):4856-4859.

35. Fam NP, Ho EC, Zahrani M, Samargandy S, Connelly KA. Transcatheter tricuspid valve repair with the PASCAL system. JACC Cardiovasc Interv. 2018;11(4):407-
408.

36. Hahn RT, George I, Kodali SK, Nazif T, Khalique OK, Akkoc D, Kantor A, et al. Early single-site experience with transcatheter tricuspid valve replacement. JACC Cardiovasc Imaging. 2019;12(3):416-429.

37. Taramasso M, Hahn RT, Alessandrini H, Latib A, Attinger-Toller A, Braun D, Brochet E, et al. The International Multicenter TriValve Registry: which patients are undergoing transcatheter tricuspid repair? JACC Cardiovasc Interv. 2017;10(19):1982-1990.

38. Taramasso M, Alessandrini H, Latib A, Asami M, Attinger-Toller A, Biasco L, Braun D, et al. Outcomes after current transcatheter tricuspid valve intervention: mid-term results from the International TriValve Registry. JACC Cardiovasc Interv. 2019;12(2):155-165.

39. McElhinney DB, Cabalka AK, Aboulhosn JA, Eicken A, Boudjemline Y, Schubert S, Himbert D, et al. Transcatheter tricuspid valve-in-valve implantation for the treatment of dysfunctional surgical bioprosthetic valves: an international, multicenter registry study. Circulation. 2016;133(16):1582-1593. 